\title{
Erratum: Measures of electronic-vibrational entanglement and quantum coherence in a molecular system [Phys. Rev. A 92, 042323 (2015)]
}

\author{
Mihaela Vatasescu \\ (Received 10 June 2016; published 24 June 2016)
}

DOI: 10.1103/PhysRevA.93.069906

In the original paper, Eq. (38) should be replaced by

$$
\mathcal{I}_{S}\left(\hat{\rho}_{e l}, \hat{H}_{e l}\right)=\frac{1}{1+\sqrt{2 L(t)}}\left[V_{g}(R)-V_{e}(R)\right]^{2}\left|\left\langle\psi_{g}(R, t) \mid \psi_{e}(R, t)\right\rangle\right|^{2},
$$

where $L(t)$ is the linear entropy of entanglement given by Eq. (11). As a consequence, Eq. (39) takes the form

$$
\mathcal{I}_{S}\left(\hat{\rho}_{e l}, \hat{H}_{e l}\right)=\frac{1}{4[1+\sqrt{2 L(t)}]}\left[V_{g}(R)-V_{e}(R)\right]^{2}\left[C_{l_{1}}\left(\hat{\rho}_{e l}\right)\right]^{2} .
$$

Additionally, Eq. (43) should be corrected to

$$
\mathcal{I}_{S}\left(\hat{\rho}_{e l, v i b}, \hat{H}_{e l} \otimes \hat{I}_{v}\right)=\left[V_{g}(R)-V_{e}(R)\right]^{2} P_{g}(t) P_{e}(t) .
$$

The above-mentioned modifications of Eqs. (38) and (43) entail a new form of Eq. (44), which shows the connection (established in the $2 \times N_{v}$ case) between the linear entropy of entanglement $L(t)$ and the two types of skew information corresponding to the electronic Hamiltonian. Consequently, Eq. (44) should be replaced by the following:

$$
\mathcal{I}_{S}\left(\hat{\rho}_{e l, v i b}, \hat{H}_{e l} \otimes \hat{I}_{v}\right)-[1+\sqrt{2 L(t)}] \mathcal{I}_{S}\left(\hat{\rho}_{e l}, \hat{H}_{e l}\right)=\frac{1}{2}\left[V_{g}(R)-V_{e}(R)\right]^{2} L(t) .
$$

Moreover, Eqs. (47) and (48), respectively, must be replaced by

$$
\begin{gathered}
\mathcal{I}_{S}\left(\hat{\rho}_{e l, v i b}, \hat{H}_{e l} \otimes \hat{I}_{v}\right)=\sum_{\alpha, \beta, \alpha \neq \beta}^{N_{e l}}\left[V_{\alpha}(R)-V_{\beta}(R)\right]^{2} P_{\alpha}(t) P_{\beta}(t), \\
\mathcal{I}_{S}\left(\hat{\rho}_{e l}, \hat{H}_{e l}\right)=\sum_{\alpha, \beta, \alpha \neq \beta}^{N_{e l}}\left[V_{\alpha}(R)-V_{\beta}(R)\right]^{2}\left|\left\langle\alpha\left|\sqrt{\hat{\rho}_{e l}}\right| \beta\right\rangle\right|^{2} .
\end{gathered}
$$

The corrected form of Eq. (48) reinforces the observation made in the article about the complexity of quantum correlations for more than two electronic states. The paragraph following Eq. (48) in the main paper should be partially amended as follows: "the difference $\mathcal{I}_{S}\left(\hat{\rho}_{e l, v i b}, \hat{H}_{e l} \otimes \hat{I}_{v}\right)-\mathcal{I}_{S}\left(\hat{\rho}_{e l}, \hat{H}_{e l}\right)$ is a sum containing correlations terms of the type $\left[P_{\alpha}(t) P_{\beta}(t)-\right.$ $\left.\left|\left\langle\alpha\left|\sqrt{\hat{\rho}_{e l}}\right| \beta\right\rangle\right|^{2}\right]$ as significant quantities, whereas the linear entropy $L(t)$ expressed in Eq. (15) is a sum containing terms $\left[P_{\alpha}(t) P_{\beta}(t)-\left|\left\langle\psi_{\alpha}(R, t) \mid \psi_{\beta}(R, t)\right\rangle\right|^{2}\right] . "$

These corrections do not affect any other part of the paper, leaving unchanged our discussion and the overall conclusions. 